\section{A new tool for cloning and gene expression in Streptococcus pneumoniae}

Susana DOMINGUES ${ }^{(1)}$, Andreia Cunha AIRES ${ }^{(1)}$, Mari Luz MOHEDANO ${ }^{(2)}$, Paloma LÓPEZ ${ }^{(2)}$ and Cecília Maria ARRAIANO ${ }^{(1)}$

1. Instituto de Tecnologia Química e Biológica, Universidade Nova de Lisboa, Av. da República, 2780-157 Oeiras, Portugal.

Microbiología Molecular y Biología de las Infecciones, Centro de Investigaciones Biológicas CIB (CSIC), 28040 Madrid, Spain.

Contact name: Susana Domingues (susanadomingues@itgb.unl.pt)

\section{ABSTRACT:}

A new replicon suitable for cloning and gene expression was successfully introduced into Streptococcus pneumoniae. The non-integrative lactococcal vectors pIL253 (high-copy) and pIL252 (low-copy), which are based on the promiscuous theta-replicating plasmid pAM $\beta 1$, were established in pneumococcus. The stability and the small size of these plasmids, together with the presence of a helpful multi-cloning site make them a useful genetic tool for gene expression in this bacterium. The functionality of the system was tested by cloning and expressing the pneumococcal RNase $\mathrm{R}$ in pIL253. Full constitutive expression of the cloned gene was observed, clearly demonstrating that this plasmid can be used as an expression vector in S. pneumoniae. Moreover, gene expression can be regulated by the use of the low- or high-copy vector versions. The existence of other replicative plasmids based on this family, which are also probably functiona in pneumococcus, further brondens pIL252 or pIL253 together with the pLS1 plasmid, a pMV158 derivative, which replicates via rolling circle mechanism. This fact greatly increases the ability to manipulate this bacterium.

The availability of a novel family of replicative vectors for genetic manipulation in S. pneumoniae is an important contribution to the study of this pathogenic microorganism.

Keywords: pneumococcal cloning, expression vector, replicating plasmid, non-integrative vector

\section{Introduction}

Non-integrative plasmid vectors are very scarce in S. pneumoniae and most of the studies have been carried out on the broad host range, rolling circle replicating (RCR) plasmid pMV158 [1] and on its derivative, pLS1 [2]. However, most of the pLS1 derivatives are large plasmids that are not easy to handle. Most of them lack a useful multi-cloning site and the respective sequence is often not available.

These observations prompted us to search for cloning vectors based on another replicon that could be suitable for the expression of genes in $S$. pneumoniae. pIL253 is a pAM $\beta 1$-based plasmid, a well characterized theta-replicating promiscuous replicon isolated from Enterococcus faecalis [3]. Plasmids belonging to the pIL253 family have been extensively used for gene cloning and expression in Gram-positive bacteria. This is mainly due to their small size $(<5 \mathrm{~kb})$ and stability, the presence of a useful multi-cloning site (MCS), sequence availability and their demonstrated ability to stably maintain large DNA inserts in L. lactis [4] Furthermore they are available in low- (pIL252) or high- (pIL253) copy number versions [4], which allows a better control of gene expression.

We show that the pIL252/pIL253 vector family autonomously replicates and is stably maintained in S. pneumoniae, and demonstrate the utility of this plasmid family as pneumococcal expression vectors. Furthermore, these vectors are compatible with pLS1, which is undoubtedly one of the most useful properties of these plasmids.

\section{Results}

2.1. pIL253 autonomously replicates in $S$. pneumoniae

pIL253 was isolated from exponentially growing S. pneumoniae TIGR4 erythromycin resistant transformants (Fig. 1) indicating that this plasmid is able to autonomously replicate in pneumococcus. The same electrophoretic mobility forms as those of the original $L$. lactis preparation were observed. pIL253 therefore seems to be structurally stable in $S$. pneumoniae and it can be stably maintained under selective pressure.

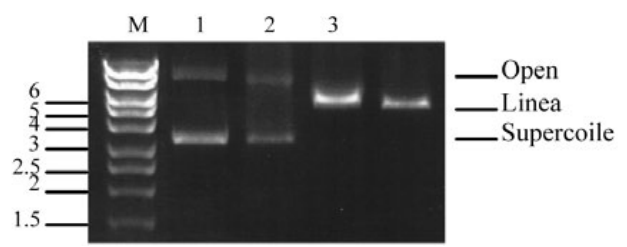

Fig. 1: Structural stability of pIL253. 1 -Original pIL253 preparation; 2- pIL253 extracted from S. pneumoniae Original pIL253 prop digested With $P$. 253 prep digested with $P$ st 1.

An analogous result was observed for pIL252, a low copy version of pIL253 (data not shown).

2.2. pIL252/pIL253 compatibility with p LS

We tested if pIL252/pIL253 could be maintained in $S$. pneumoniae together with $\mathrm{pLS} 1$. Plasmids extracted from exponentially growing single transformants (S. pneumoniae TIGR4 cells carrying pLS1, pIL252 or pIL253) were compared with those extracted from double transformants (pLS1/ pIL252 or pLS1/pIL252) by restriction analysis. The result obtained with pIL252 shows that none of the plasmids underwent structural rearrangements (Fig. 2). An analogous result was obtained when pIL253 was used instead (data not shown). Thus, pneumococcal cells are able to stably maintain both pIL252/pIL253 vectors and pLS1.

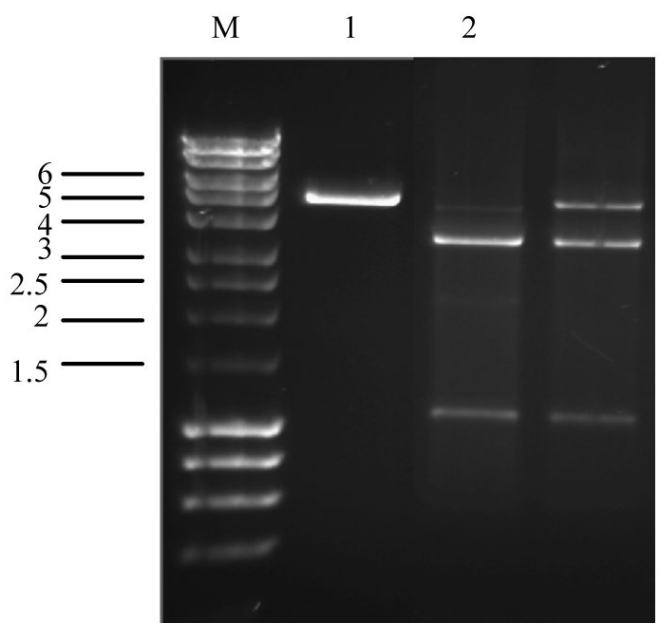
Fig. 2: Compatibility of pIL252 with pLS1. Digestion of
plasmid preparations with PstI 1-pIL252; 2- pLS1; 3$p I L 252+p L S 1$.

\subsection{Gene cloning and expression from pIL253}

RNase R expression from pIL253 was evaluated by RT-PCR and Western blotting. The results in Fig. 3 show that RNase R is highly expressed from pIL253. We also observe that expression from this plasmid seems to be constitutive and does not change with temperature. Full detection of RNase $\mathrm{R}$ in late exponential cultures indicates that the DNA insert remains stable after several generations. These results clearly demonstrate that the lactococcal vector pIL253 is suitable for gene cloning and expression in S. pneumoniae.

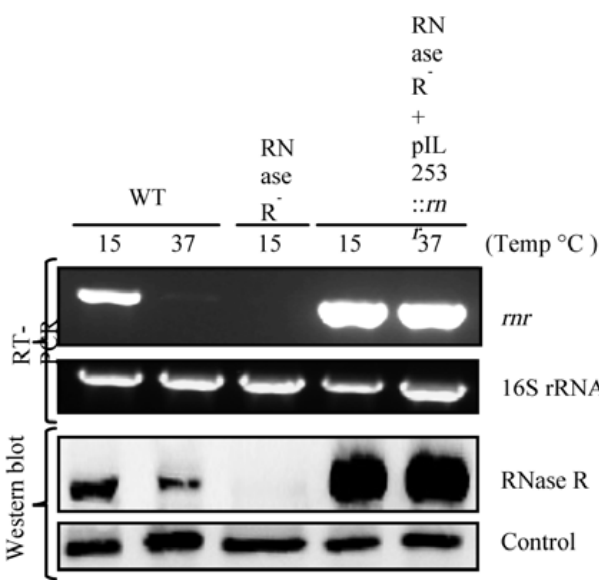

Fig. 3: Expression of pneumococcal RNase $R$ from pIL253. 


\section{Conclusion}

The pIL253 vector, a pAM $\beta 1$ derivative, was successfully used for cloning and expression of a pneumococcal chromosomal gene in host cells of this species. Moreover, this small sized vector contains a functional multi-cloning site with several unique restriction sites and it is able to stably miche able to stably maintain large DNA inserts. The possibility of using the low-copy number version (pIL252) offers another option for the genetic manipulation of pneumococcus. Moreover, the availability of other plasmid refined tools constructed on the basis of this family with different cloning possibilities constitutes an extra advantage. Besides pIL253 and pIL252, pIL871, a chloramphenicol resistance derivative of pIL253 was also constructed [5]. pIL253 and pIL871 share the same replication protein and can be stably maintained in a mixture by double selection with erythromycin and chloramphenicol in L. lactis [5]. We have also shown that $\mathrm{pIL} 252 / \mathrm{pIL} 253$ can be maintained in $S$. pneumonia simultaneously with pLS1. This result indicates that these replicons are compatible, which significantly broadens their potential applications.

\section{Acknowledgements}

This work has been sponsored by Fundação para a Ciência e a Tecno logia including grant \# PEst-OE/EQB/LA0004/2011 and the Spanish Ministry of Economy and Competitiveness grant AGL2012-40084-C03-01. Fundação para a Ciência e Tecmologia gave financial support to S. Domingues (Postcoc Aires (Fellowship)

\section{References}

[1] Vickers BURDETT, Identification of tetracyclineresistant R-plasmids in Streptococcus agalactiae (group B), Antimicrob Agents Chemother, 18, 5, 753-760, 1980

[2] Diane L. STASSI, Paloma LOPEZ, Manuel ESPINOSA, Sanford A. LACKS, Cloning of chromosomal genes in Streptococcus pneumoniae, Proc Natl Acad Sci USA, 78, 11, 7028-7032, 1981.

[3] Claude BRUAND, S. Dusko EHRLICH, Laurent JANNIERE, Unidirectional theta replication of the structurally stable Enterococcus faecalis plasmid pAMB1, Embo J, 10, 8, 2171-2177. 1991.

[4] Daniel SIMON, Alain CHOPIN, Construction of a vector plasmid family and its use for molecular cloning in Streptococcus lactis, Biochimie, 70, 4, 559-566, 1988.

[5] Susana DOMINGUES, Alain CHOPIN, S. Dusko EHRLICH, Marie-Christine CHOPIN, The Lactococcal abortive phage infection system AbiP prevents both phage DNA replication and temporal transcription switch, J Bacteriol, 186, 3, 713-721, 2004

\section{A Synthetic Biology Platform for Streptococcus pneumonia}

Robin A. SORG and Jan-Willem VEENING

Molecular Genetics Group, Groningen Biomolecular Sciences and Biotechnology Institute, Centre for Synthetic Biology, University of Groningen, Nijenborgh 7, 9747 AG, Groningen, the Netherlands.

Contact name: Robin A. Sorg (r.a.sorg@rug.nl)

\section{ABSTRACT:}

The introduction of engineering principles to molecular biology promises to represent a critical step both towards a deeper understanding of cellular mechanisms as well as to real applications by programming synthetic circuits with designed characteristics. With this study, we aim to create a genetic platform for synthetic biology in Streptococcus pneumoniae. The model organism is a very promising novel chassis for the field since it has a small genome, it becomes naturally competent for transformation, there are well established assays and it is an important human pathogen. An integration plasmid containing specific restriction sites for BioBrick cloning was created by the Golden Gate assembly method. We show that the designed plasmid offers robust and easy cloning and confers reliable expression within the pneumococcal genome. Synthetic promoter libraries were created and promoters of different strength were identified using bioluminescence assays. In addition, several new selection and counter-selection markers and inducible systems for $S$. pneumoniae were constructed and characterized. The methods developed here will help creating similar platforms of the next generation and might serve as an excellent starting point for synthetic biology research in bacterial pathogens. 